\title{
Location-based Authentication and Authorization Using Smart Phones
}

\author{
Feng Zhang, Aron Kondoro, Sead Muftic \\ Communication Systems \\ School of Information and Communication Technology \\ Royal Institute of Technology (KTH) \\ Stockholm, Sweden \\ fengz@kth.se, kondoro@kth.se, sead@kth.se
}

\begin{abstract}
Authentication and authorization are two of the most important security features for mobile transaction systems. Most commonly, these schemes depend on three factors: what you know (secret), what you have (token), and what you are (biometrics). In this paper, we propose a locationbased authentication and authorization scheme for mobile transactions using smart phones. The paper first describes the distinguished features and the architecture of our proposed solution. Second, the core of our design, including three parts: location registration, authentication and authorization as well as location verification, are described.
\end{abstract}

Keywords-location; authentication; authorization; mobile;

\section{INTRODUCTION}

Currently, one of the most common authentication mechanisms is based on the use of passwords. This is due to its ease of implementation for the Service Providers (SPs), cost effectiveness and its familiarity to end-users. However, it has also been one of the least secure methods compared to other options. People generally choose weak passwords and use the same ones for multiple services. As a result, accounts get hacked, people lose money, and privacy is breached etc. In order to counter those problems, security critical services, such as online banking, started to use multi-factor authentication solutions. For example, permanent passwords are combined with other mechanisms, such as special tokens, that can generate one-time passwords. The use of more than one factor has been observed to be more secure than depending only on a single one. Most solutions depend on factors that fall under three categories, namely: (1) what you know e.g. password, personal identity number (PIN); (2) what you have e.g. smart cards, token, etc.; and (3) what you are e.g. biometrics, such as fingerprints, voice recognition, palm scanning or retinal scans. Even though these factors are sufficient for most cases, there is still additional room for improvements and alternatives. One of these factors is user's location.

There are several existing systems that utilize location information to provide authentication and authorization solutions. However, these solutions usually require a specially designed infrastructure and special devices that can be used to determine their locations [1] [2] [3]. It is rather difficult for these solutions to get widely deployed since some special requirements have to be fulfilled.
Meanwhile, smart phones are gaining popularity all over the world, especially in the US, Europe and Asia [4-6]. Most of these smart phones are equipped with inbuilt global positioning system (GPS) chips that can accurately detect the location of the user. This is evidenced by the explosion of location-based services, such as Google Maps, Foursquare, Gowalla and Yelp. In addition, a lot of innovation is constantly occurring in the area. Companies such as Skyhook, Google and Apple continually improve their location detecting technologies by creating large databases of wireless access point and cell tower locations. The overall results have been the improvement in the accuracy of the detected locations. It is now feasible to determine the location of a user within meters of his/her actual location [7].

Based on these facts described above, the use of smart phones can be a potential solution for location-based authentication and authorization schemes. Smart phones can be used to detect and send the location of a particular user to back-end servers, which shall verify the location as a factor for authentication and authorization purpose. There are several advantages of our solution and these advantages represent the main differences between our solution and other existing solutions:

1. It is not necessary to set up a specialized infrastructure in order to use our solution. Instead, the mobile network infrastructure, which has been widely established, is used;

2. Compared with most exiting solutions, described in the related work, where special devices must be used for location-based services, in our solution users do not have to use specific devices rather than a smart phone, which they are carrying everywhere, every day;

3. It is easy and flexible to integrate our solution with any existing authentication and/or authorization systems. Our solution can work as a security plugin for existing systems;

4. These smart phones have stable platforms on which secure applications and services can be built on.

5. Smartphones possess a variety of location technologies, which enable locations of objects or people to be accurately determined within meters [17];

6. Provides application level end-to-end security for protecting location data; 
7. Multiple location sensing technologies, built in the smart phones, are used in our solution, which can provide better services, compared with other solutions where only single technology is used.

In this paper we propose a solution that uses GPS technology provided by smart phones for location-based authentication and authorization. The paper is organized as follows: in Section two we highlight and briefly describe some related work have been done in this area. In Section three, we describe the architecture and components of our solution. After that in Section four, the details of our solution, including location registration, authentication and authorization as well as location verification are described. The conclusion and future work is presented at the end.

\section{RELATED WORK}

A fair number of research studies have been performed in the area of location-based authentication and authorization. Denning and Macdorman [2] were among the first authors to perform research studies about location-based authentication and to highlight its importance for improving network security. In their paper they argued that location information can be used for both preventing network breaches and also for facilitating investigations in cases when breaches occur. In a virtual environment where physical borders are blurred, location determination during authentication can be helpful in many scenarios e.g. remote access to critical systems, authenticating financial transactions, enforcing export controls on software and so on. They describe a technology by the International Series Research in USA, called CyberLocator, which is used to achieve location-based authentication by using what is called a location signature. A client that wants to access a protected resource is challenged to provide a location signature, which is then verified by the server. The server does this by also computing its own location and comparing it to the one provided by the client. Since the location signature is unique for each location at any given time, this information cannot be spoofed or replayed later. However, in order to achieve this, CyberLocator needs its clients to possess a special kind of GPS sensor that is different to the ones that are commercially available.

YounSun et al. [1] propose a location-aware access control mechanism (LAAC) based on a WLAN infrastructure of wireless access points and wireless mobile devices, such as PDAs and wireless laptops. Access is granted to a device located inside a region formed by overlapping coverage of multiple access points. Each access point periodically broadcasts a random nonce which is captured and used by the device to generate a location key. Devices outside the range of the access points won't be able to receive these random nonces and consequently won't be able to derive valid location IDs. In this way access is granted only within specific locations.

Bao [8] proposes similar mechanism using wireless access points. His system is known as LENA (Location Enforced Network Access). LENA has two schemes, one known as LENA-SK (LENA using Security Keys) uses Diffie-Helman key exchange protocol to authenticate user location, authorize network access, and distribute a key for data encryption. The other scheme, LENA-PAP (LENA using Personal AP Protocol) uses mobility management protocol to ensure authenticity of location claims. These mechanisms are designed specifically for controlling access to wireless networks.

Jansen and Kolorev [3] designed a location-based authentication mechanism that involves policy beacons and mobile devices. These policy beacons broadcast and communicate location data to mobile devices using Bluetooth. Mobile devices determine their proximity to beacons and calculate their location relative to them. Based on this location certain functionalities in the mobile devices are enabled or disabled accordingly. Policy beacons establish a perimeter with a distinct organization policy. Devices within this perimeter inherit this policy. Their setup, however, focuses only on controlling the use of mobile devices, especially in an environment such as in an organization and it requires a significant costly infrastructure setup and synchronization of policy beacons.

Takamizawa and Kaijiri [9] proposed and designed an authentication method using location information obtained from mobile telephones that is suitable in web-based education applications. A student who wants to login into the web-based application, in addition to using username and passwords, has also to provide his/her location through a mobile telephone in order to prove the authenticity. In their method, location from a mobile phone is determined using GPS. For that, mobile phone must be equipped with a GPS receiver and a clear view of the sky is needed for the process to work. QR codes are also used for web applications to prompt the mobile phone for the location. The user has to scan the code from the screen using his/her mobile phone and therefore a phone needs a camera. In addition, the authors did not pay attention to security threats and vulnerabilities for their location-based authentication method and as such the mechanism may be susceptible to trivial attacks. For example, the location could be easily spoofed or modified.

Ardagna et al. [10] analyzed how location information can be used to strengthen access control mechanisms by adding features for defining and enforcing location-based policies. They proposed design of a Location-based Access Control (LBAC) architecture and provided an extension to the XAMCL policy language (introduced by the Open Geospatial Consortium - OGC) for defining and describing geographic location coordinates. This extension is known as GEOXAMCL. They showed examples of how this can be used to express access control rules that can be used in a typical application.

As described above, some of the existing solutions have been focused on designing and constructing general conceptual security models for these kinds of mechanisms. Some have demonstrated and justified the use of location in improving existing security mechanisms. Despite the security features that they offer, most of these solutions however have suffered from problems such as practicality, usability, reliability and cost. In addition since the location signature depends on GPS, the mechanism suffers a lot of reliability issues especially indoors or in places where there 
is no clear view of the sky. Others have proposed protocols, which however apply only to specific scenarios or require specific devices. A general and flexible approach that can be applied in different situations is still lacking.

A method that takes advantage of the recent advancement in location-sensing technologies, especially provided by smart phones, is needed. These advancements have led to the improvement and reliability of location information and thus rendering it more useful. Earlier approaches have relied on technologies, once considered state-of-the-art, but currently either obsolete or mainly not adequate. In addition, these approaches require extensive user involvement making them less user-friendly. With the current technology it is possible to make this technology transparent and convenient for users.

\section{ARCHITECTURE AND COMPONENTS}

Our proposed solution comprises six components, which are combination of various servers and applications:

- Location-based ID (LBID) server - is the core component of our solution, which stores users' location information and authorization policies. It provides location registration, authentication and authorization services;

- Certificate Authority (CA) server - provides certification of all system participants, issuing, managing and distributing their certificates;

- Authentication server - provides authentication service for all participants. It can be any existing authentication service, such as username-password authentication. And it is extended to connect with the LBID server to provide location-based authentication service;

- Authorization server - provides authorization services for all participants. It can be any existing authorization service, such as role-based authorization. And it is extended to connect with the LBID server to provide location-based authorization service;

- Service Provider (SP) server - provides various mobile services; and

- Location-based Client (LBC) Application - an application running on user's mobile device, capable to collect location information from trusted Location Providers (LP) and providing user interfaces to register, store and manage location data.

The architecture and components are shown in Figure 1. System setup is performed by system administrators, establishing location-based authorization policies and certifying all system components. Users then use LBC to interact with LBID server and Authorization server. After valid and successful registration of their current location, users submit access request to Authentication server, which interacts with LBID server and Authorization server to evaluate the request based on the information registered in LBID server and on authorization policies registered in the Authorization server. The result is sent to the targeted SP server, which decides accordingly to either allow or deny access request.
One of the most important concerns of location-based services is security and privacy of the location information.

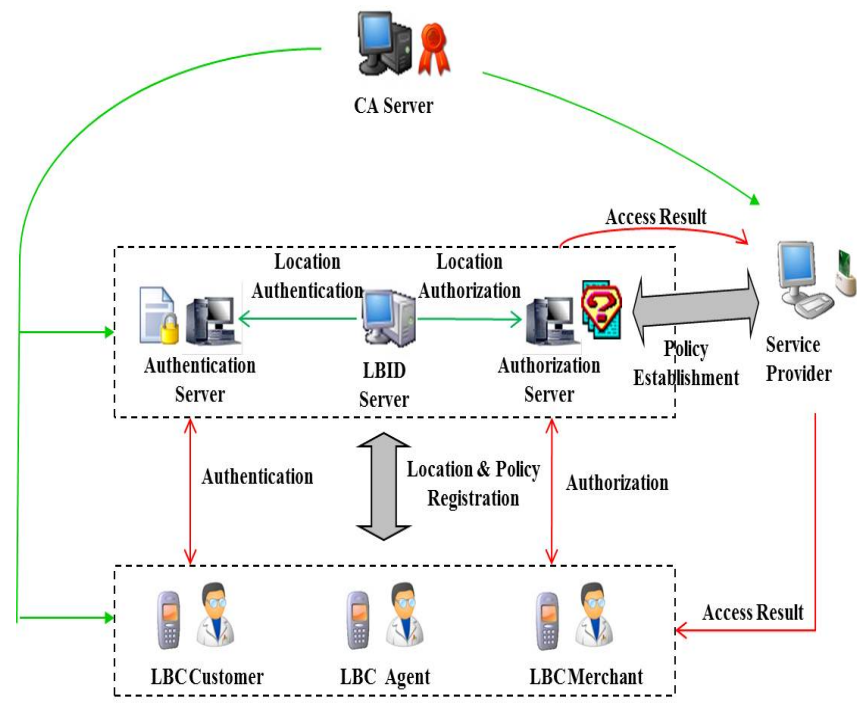

Figure 1. Location-based authentication and authorization architecture

In order to protect location information and the privacy of user's location, we use cryptographic techniques, based on Public Key Infrastructure (PKI) and certificate mechanisms. The details of certification issuance and distribution in a mobile environment are not described in this paper. It is assumed that every entity has already received certificate. All certificates are issued by CA server in a standard way. In that way, the integrity of location data sent from LBC to LBID server can be verified. All the messages exchanged between all components are digitally signed by message initiator.

\section{PROTOCOLS}

Two protocols are designed for our system: registration protocol and authentication and authorization protocol. Registration is performed only once, after joining the system. Authentication is performed at the beginning of each session, based on principles of single sign-on protocol. Authorization is enforces for every service request.

In our solution, two types of location information are used: static location and dynamic location. Static location refers to the fixed location captured at the time of registration and stored in the database on LBID server for future comparison during authentication and authorization. Static location usually remains the same until it is explicitly changed by the user. Dynamic location refers to the location information captured every time when user requests access to some resources or mobile services. Dynamic location always changes based on the user's movement and current location.

The location information (both dynamic and static) that is captured by the LBC and sent to the server comprises four parameters: longitude, latitude, range of location accuracy and the underlying technology that was used to determine the location. In addition, the static location also contains the user 
specified location range that specifies the area in which the user can authenticate successfully. The longitude and latitude are used to identify a specific precisely a geography location. Every location estimate contains a level of uncertainty as the result of the location sensing technology used. The range of location accuracy is used to express this uncertainty to the server in order to facilitate in making valid authentication and authorization decisions.

\section{A. Registration}

As the first step, users must be registered before accessing resources or services. In that process, user's identity information, location information, and authorization policies must all be registered. Registration is performed initially only once for new users. In this phase, user registration data, together with his/her current location is collected and stored in the LBID server.

Before the process begins, LBC must be downloaded and installed on the user's mobile device. It comes preloaded with the trusted Root CA certificate. During its activation, as the first step, user specifies his/her PIN, which is used as local authentication credential and at the same time as a seed to generate local secret key, used to encrypt LBC's private key and all other locally-stored data. Then, user's identity information e.g. user name, mobile number etc. are entered and sent to the LBID server. After that, LBC generates a key pair, and sends certificate request to the CA server. In the reply, it receives and stores locally two certificates: its own certificate, and the certificate of the LBID server. The Root CA certificate is used to validate these two received certificates.

After registration of identity data and obtaining the certificate, as the final step in this process, user registers his/her static location. For that, user first sends locationregistration request to the LBID server. The request is digitally signed by the LBC and accompanied by user's certificate. Upon receipt and successful verification of user's certificate and the request, LBID server generates a random number, associated with the received information, and sends it back to the user.

User activates location-registration function of the LBC by entering correct PIN and then is required to enter the random number received from LBID server. The random number is used to prevent replay attack. The LBC captures user's current location and sends it together with the random number to the LBID server. The location information is signed by user's private key and encrypted using LBID server's public key. When LBID server receives the data, it first matches the received location information with the previously received user's registration request using the included random number. Then, LBID server decrypts user's location information using its private key and verifies user's signature with user's certificate and stores user's location information together with user's identification data.

After completion of both steps, user's registration information (identification and static location) is stored in the database and results are sent back to the $\mathrm{LBC}$, which then presents a confirmation message to the user. Figure 2 shows the overview of the registration process.
User (and SP) then can update their authorization entries in the authorization policy based on their location parameters. The policy is stored at the LBID server. Thus, our solution provides location-based authorization in addition to the existing authorization mechanism. For example, user may register in a policy location-based rule enforcing that the payment with the amount higher than $\$ 100$ must be done from his/her home location. The registration and updates of policy entries works as follows: user (or SP administrator) enters policy rule using LBC. The rule contains mainly three fields:

a. Subject - the entity who accesses the resources

b. Object - the resources to be accessed

c. Action - the way that the subject accesses the object

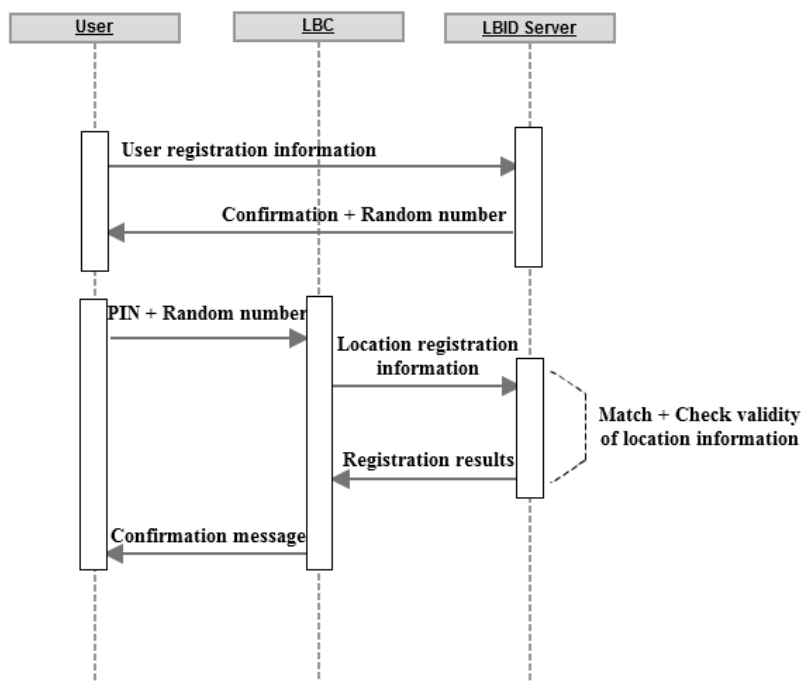

Figure 2. Location registration

For example, in the case mentioned above, the subject is user, the object is user's account, and the action is to pay. After entering data for a policy rule, the LBC sends data together with user's location data, determined in that moment to the LBID server. The message is signed by LBC and encrypted with LBID server's public key. Once receiving the message, LBID server decrypts it with its private key and verifies user's signature with user's certificate and stores the information in form of a new rule in the authorization policy.

\section{B. Authentication and Authorization}

This is the second phase when the actual location-based authentication and authorization process is performed. The protocol is performed every time when the user requests access to some resources or service from the system. Our solution can be integrated with any existing authentication and authorization systems. In this case, there are authentication server and authorization server providing the existing authentication and authorization service. Our proposed solution can be integrated with them.

The process begins when the user tries to access the protected resource (e.g. login into his/her account). The process is initiated by user sending a service request to the 
SP server. SP server directs service request to the Authentication server to authenticate the user. The Authentication server sends an authentication challenge to the user. The user then responds to the challenge by providing his/her security credentials back to the Authentication server for the authentication purpose, which can be any existing mechanism depending on the implementation of the particular system. For example, it can be a username/password, one-time password (OTP) or certificate-based challenge/response authentication.

Upon successful verification of user credentials, Authentication server sends a location verification request to the LBC running on user's smart phone. The LBC prompts the user to enter PIN. User responds by entering the PIN in order to authorize a location response. LBC then determines user's location and sends it back to the authentication server within location response message. The location information is signed by LBC's private key and encrypted by LBID server's public key.

After that, the authentication server delivers user's location information to the LBID server. LBID server decrypts the message using its private key and verifies user's digital signature. If the verification is OK, it compares user's location information with the location data stored in its database during registration phase, namely compare static location with dynamic location, and sends authentication result with user's location information to the authentication server. If the authentication succeeds, authentication server sends authorization request, comprising user's access request and user's location information, to the authorization server. Authorization server first performs existing authorization mechanisms, such as role-based or attribute-based authorization, based on the locally stored policies. After that, authorization server sends location-based authorization request, which comprises subject, object, action and user's location to LBID server. LBID server decrypts the message using its private key and verifies user's digital signature. If the verification is $\mathrm{OK}$, it compares user's access request and location information with entries stored in the location-

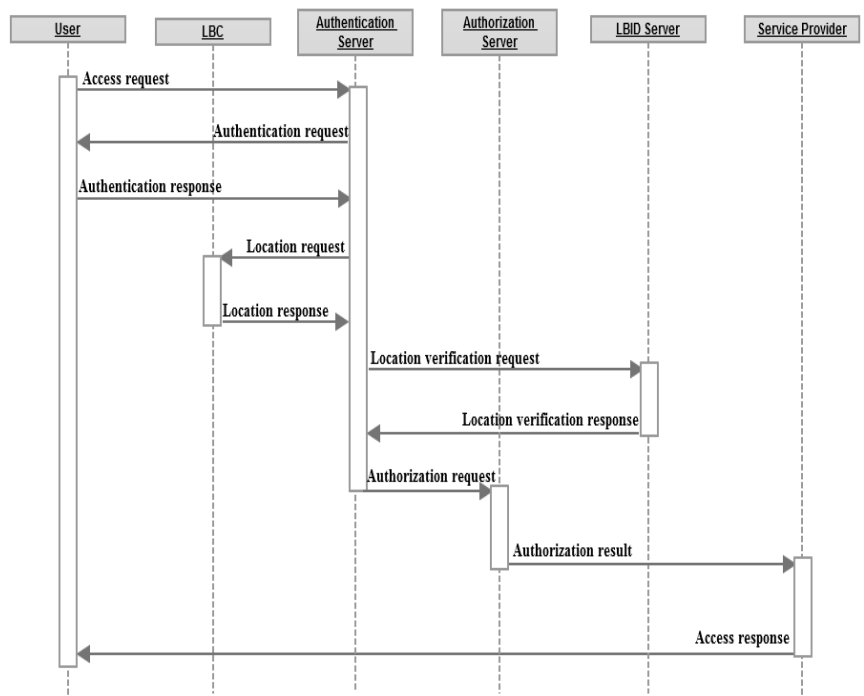

Figure 3. Location-based authentication and authorization authorization policy and sends authorization result to the authorization server. Authorization server finally notifies SP server the authorization result. SP server decides whether to approve user's service request based on the authorization result. Figure 3 shows the sequence of steps involved in the location-based authentication and authorization protocol.

\section{Location Verification}

One of the most crucial steps in a location-based authentication and authorization mechanism is the verification of the location claim provided by the user. The security of the whole systems can either succeed or fail depending on the effectiveness of this step. The decision whether a user is authenticated or not depends on the validity of his/her presented location. When a user authenticates to the system he/she presents his/her location, which has been captured and calculated by the location sensing client. The verification algorithm then has the responsibility to check this location claim, verify its validity, compare it to specified authorized locations and make a decision whether the user is authenticated or not. The goal of this process is to prevent location spoofing and make sure that the clients are really in the locations that they claim to be at.

There are several ways in which the location provided by a smartphone can be spoofed in order to fool a locationbased security system [11]. These attacks can happen on different levels of the smartphone platform stack. As such they can be categorized as follows:

\section{- Spoofing on the hardware level}

This is done via the location (GPS) module. An attacker can directly hack into the GPS hardware or module or simulate it in software and modify it to provide fake location signals to the smartphone operating system and consequently to the location based client. In this way, the signals are intercepted at the lowest possible level before they reach the client and as a result it ends up deducing an incorrect location.

\section{- Spoofing on the OS level}

This is done by intercepting and modifying the location APIs in the smartphone operating system so that they report a fake location to the client application. This can be achieved by modifying or running a modified version of an operating system which has been programmed to report the locations that an attacker desires. This kind of an attack is possible in open source type of smartphone OSs where the source code is accessible and can be modified.

\section{- Spoofing on the application level}

This kind of spoofing happens directly on the location client application by modifying its source code or by intercepting and modifying the final location result that is sent to the location-based server. A fake or modified copy of the location client application can be installed in the smartphone and report spoofed locations to the server. In addition, the location results from a valid client can also be intercepted in transit and modified to desired values before they reach the server.

Another interesting attack [12] that targets location services in smartphones works by simulating certain environmental parameters i.e. access points, cell towers in 
order to fool the location modules that depend on this information to deduce the location of a device. This kind of attack however is not reliable and requires a lot of effort to carry out especially in a global scale.

A number of different techniques can be used for location verification in order to mitigate the mentioned methods of location spoofing. One is distance bounding [13]. This kind of technique takes advantage of the physical limitations of wireless technologies to deduce and verify the location of a particular client. For example, a nearby Wi-Fi access point can collaborate with the location client in a smartphone to verify that the phone is in a location that it claims to be in. Another approach is to use IP addresses to geo-locate the client that is communicating with the server. Some research has shown that IP addresses can be used to roughly deduce the general location of a client [14]. These IP addresses are issued by mobile network operator (MNO) and cannot be easily changed by the client. Therefore, it is more difficult to spoof these IP addresses, which makes the approach more secure. This approach also has some disadvantages, including the frequent changing of client IP addresses, the level of accuracy of the location estimates and the use of proxies/gateways which may hide true locations of clients. Another location verification technique is based on network latency measurements [15]. By comparing the latency (round-trip) time to a client with other known reference points, a locating server can use this technique to verify the location of a particular client. All of these approaches individually have their strengths and weaknesses.

Our location verification mechanism solves these problems by employing a hybrid approach. It combines different techniques and takes advantage of their strengths in order to verify the location of sufficiently with enough confidence. The approach can be considered as a "strengthin-depth" method, in which different individual methods complement each other and act as a layer of verification steps, where if one method is fooled the others can detect and prevent spoofing.

The location verification mechanism takes into account the following parameters:

1. Two sets of location coordinates from two different location sources;

2. IP address of the client (smartphone);

3. MAC (Media Access Control) address of a nearby access point with the strongest signal.

\section{1) Registration}

During the registration process the location information of the user is collected by the LBC running in the smartphone, sent to the server and stored in the database. This information includes the latitude (LAT), longitude (LNG) and accuracy (in a certain unit of measurement e.g. meters) as obtained from the location API of the smartphone. As shown in Figure 4, it represents the exact location and a range that the user wants to signify as an authorized location, from which access can be granted.

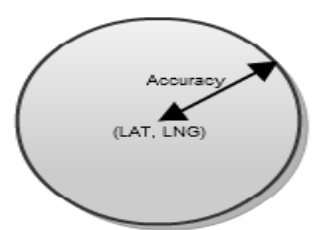

Figure 4. Location information

In addition, the MAC address of the strongest nearby WiFi access point is detected and stored in the database as an additional parameter. This value is obtained by comparing the RSSI (Received signal strength indicator) of all detected Wi-Fi signals and choosing the one with the highest signal strength.

\begin{tabular}{|c|c|c|c|}
\hline 159.333298 & -77.128304 & $45 \mathrm{Wi}-\mathrm{Fi}$ & 70 00:08:C7:1B:8C:02 \\
\hline 259.370136 & 18.066193 & 311 Cell Tower & 500 00:08:c7:16:8c:02 \\
\hline 538.975956 & -77.128304 & 21 GPS & 50 00:00:c0:88:0a:2e \\
\hline 659.323296 & 18.067360 & 9 GPS & 20 00:00:6b:71:1a:6a \\
\hline
\end{tabular}

Figure 5. Example of location database scheme

All of these values are then stored in the database as part of the user registration information. Figure 5 below shows an example of location database scheme.

\section{2) Authentication and authorization}

During the authentication and authorization stage, the location of the user is detected and verified by the LBID server using the following mechanism:

\section{Step 1:}

In most of the major smartphone platforms there is usually more than one source (APIs) for obtaining location. For example, on Android there is the normal Android Location API, Skyhook, etc. This is the same for iOS, Blackberry and others. Based on this, our location verification utilizes two sets of location coordinates (LAT, LNG and accuracy) that are obtained from two different location APIs. In this way, the reliability and accuracy of the location is ensured since the result does not depend only on one source.

These two location coordinates are then compared to see if the location areas they represent overlap. In normal circumstances these two areas should always overlap, as shown in the left part of the following Figure 6. If there is no overlap at all between these two location areas, as shown in the right part of the following Figure 6, it is a good indication that one or both of the sources of location are not correct and may have been compromised through one of the methods described previously. 

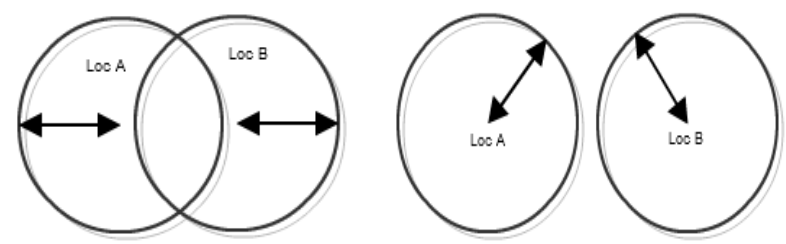

Figure 6. Step 1 of location verification

If there is no overlap location, the verification fails and the process stops. If the two results overlap, the process continues to step 2.

Step 2:

The public IP address of the client as observed by the server is recorded and used to estimate the location coordinates (LAT, LNG and accuracy) of the user using IP2Location service [16]. The result of this is then compared with both sets of coordinates from Step 1 to see if location areas are contained within the area represented by the coordinates from Step 2.

Both location areas, described in Step 1 should be contained within the location area that is calculated based on the IP address, as shown in Figure 7. The location area obtained from IP address is usually of lesser granularity which covers a bigger area and should contain both locations described in Step 1, which are more precise with smaller areas. If both locations coordinates are not contained within the larger range of area from the IP address then it is good indication that one or both of the location coordinates from Step 1 are inaccurate and may have been spoofed.

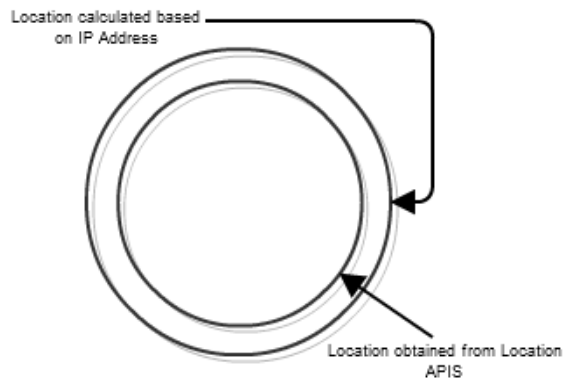

Figure 7. Step 2 of location verification

Therefore, the location verification process fails and stops if both locations from Step 1 are not contained in location from Step 2. If this check is successful the process continues to the final Step 3.

Step 3:

The MAC address of the access point, with the strongest detected Wi-Fi signal is captured and compared to the one saved during the registration process. If the two values do not match the verification process fails and stops. On the other hand if the values match, the verification process succeeds and becomes completed.

These three steps provide a series of checks to ensure that user's location as detected and reported by the LBC is correct and has not been spoofed or tampered with. After all the steps have been completed successfully, there is a high confidence in the validity of the location reported by the user.

The most accurate location result from Step 1 - the one with the highest accuracy (small range) represents the location of the user and thus is used to make further locationbased authorization decisions depending on the authorized location registered by the user.

\section{CONCLUSION}

In this paper a location-based authentication and authorization mechanism using smart phones is proposed and described. The proposed solution provides comprehensive protections for transmission, procession and verification of location information. For location verification, we propose a hybrid approach, which combines various technologies. This approach improves the confidence of verification results, compared with other solutions where only one factor is used for location verification. As a result, our location-based authentication and authorization mechanism becomes more secure and valid.

The use of location however is just the first step in using contextual information for improving security mechanism. As the smartphone technology progresses more and more sensors are integrated into the devices e.g. Proximity sensors, near field communication (NFC) and so on. This offers even further opportunities to capture and include this information about the user's environment on top of his/her location.

\section{REFERENCES}

[1] YounSun Gho, L. Bao, M.T. Goodrich, "LAAC: A Location-Aware Access Control Protocol", Mobiquitous, Third Annual International Conference on Mobile and Ubiquitous Systems, Networking, and Services, pp.1-7, 2006

[2] Denning, D. and Macdoran, P., "Location-based Authentication: Grounding Cyberspace for better Security", Computer Fraud \& Security, 1996(2), pp.12-16.

[3] Jansen, W. \& Korolev, V., "A Location-Based Mechanism for Mobile Device Security", in WRI World Congress on Computer Science and Information Engineering, Los Angeles, California USA, pp. 99-104, 2009

[4] Anon, "40 Percent of U.S. Mobile Users Own Smartphones; 40 Percent are Android | Nielsen Wire". Available at: http://blog.nielsen.com/nielsenwire/online_mobile/40-percent-of-u-smobile-users-own-smartphones-40-percent-are-android/ [Accessed September 19, 2011].

[5] Anon, "Gartner Says Sales of Mobile Devices in Second Quarter of 2011 Grew 16.5 Percent Year-on-Year; Smartphone Sales Grew 74 Percent". Available at: http://www.gartner.com/it/page.jsp?id=1764714 [Accessed September 19, 2011].

[6] Anon, "IMS Research - Electronics market research \& consultancy". Available at: http://imsresearch.com/pressrelease/Global_Smartphones_Sales_Will_Top_420_Million_Devices in_2011_Taking_28_Percent_of_all_Handsets_According_to_IMS Research [Accessed September 19, 2011].

[7] S. von Watzdorf and F. Michahelles, "Accuracy of positioning data on smartphones," 2010, pp. 1-4.

[8] Bao, L., "Location Authentication Methods for Wireless Network Access Control", in IEEE International Performance Computing and Communications Conference (IPCCC), Austin, TX, USA, pp. 160167, 2008. 
[9] Takamizawa, H. \& Kaijiri, K., "A Web Authentication System using Location Information from Mobile Telephones", Proceedings of the IASTED International Conference Web-based Education (WBE 2009)

[10] Ardagna, C.A., Cremonini, M., Capitani di Vimercati, S., Samarati, P., 2009. Access Control in Location-Based Services, in: Bettini, C., Jajodia, S., Samarati, P., Wang, X.S. (Eds.), Privacy in LocationBased Applications. Springer Berlin Heidelberg, Berlin, Heidelberg, pp. 106-126.

[11] He, W., Liu, X., and Ren, M. Location Cheating: A Security Challenge to Location-based Social Network Services. In Proceedings of CoRR. 2011.

[12] Anon, Location Spoofing Attacks on the iPhone and iPod. Available at: http://www.syssec.ch/press/location-spoofing-attacks-on-theiphone-and-ipod [Accessed November 15, 2011].

[13] J. Chiang, J. Haas, and Y. Hu, "Secure and precise location verification using distance bounding and simultaneous multilateration," in Proceedings of the second ACM conference on Wireless network security. ACM, 2009, pp. 181-192.

[14] M. Balakrishnan, I. Mohomed, and V. Ramasubramanian, "Where's that phone?: geolocating IP addresses on 3G networks," in Proceedings of the 9th ACM SIGCOMM conference on Internet measurement conference. ACM, 2009, pp. 294-300

[15] KATZ-BASSET, E.,JOHN,J., KRISHNAMURTHY,A., WETHERALL, D., ANDERSON, T., AND CHAWATHE, Y. Towards IP geolocation using delay and topology mesurements. In Proceedings of the ACM SIGCOMM Internet Measurement Conference (October 2006).

[16] Anon, IP Address Geolocation to Identify Website Visitor's Geographical Location. Available at: http://www.ip2location.com/ [Accessed October 31, 2011].

[17] Von Watzdorf, S., Michahelles, F., 2010. Accuracy of positioning data on smartphones. ACM Press, pp. 1-4. 